\title{
CRISIS Y REESTRUCTURACIÓN EMPRESARIAL
}

\author{
CPC.MIGUEL N. DÍAZ INCHICAQUI(*)
}

\section{INTRODUCCIÓN}

En nuestro país todos los días enfrentamos, frecuentemente, crisis de diversas indoles, tales como: la crisis política, social, moral, jurídico, natural y económica. En este ambiente de crisis las empresas peruanas no han evolucionado ni financiera ni económicamente y menos han alcanzado niveles competitivos para incursionar en los mercados externos; por el contrario, estas crisis nan incrementado los riesgos en los negocios que los han afectado a través de varias Jécadas y los han conducido por etapas de solvencia empresarial, en algunos casos, y en otros de insolvencia ya que los factores mencionados no pueden ser contrarrestados con solo implementar una política empresarial eficaz y eficiente.

El fracaso de las empresas peruanas bedece a un conjunto de causas complejas múltiples que pueden ser imputados por _na parte a los modelos de constitución y manejo de empresas familiares; comporzamientos específicos de sus directores, socios y participantes; a la flexibilidad y ampliación del mecanismo jurídico implementado por los gobernantes de turno y a la fropia estrategia de la empresa, al no percibir el grado de turbulencia del ciclo económico empresarial en la toma de decisiones.

Analizar La Crisis y la Reestructuración Empresarial no es estudiar sólo las causas más gravitantes de la crisis de los negocios en nuestra realidad, tales como: la falta de diversificación de los productos y servicios que se ofrecen, cambios inesperados en la demanda del mercado, atraso en los aspectos de tecnología usados, competencia desleal creada por mercados informales en el mercado nacional, carencia de recursos financieros y falta de competitividad para los mercados externos. También, debemos considerar la notoria inadaptación del medio ambiente interno de la empresa peruana a su medio ambiente externo, ya que las gerencias y sus asesores no han desarrollado estrategias que protejan y minimicen los riesgos de negocios que vienen cambiando constantemente en el medio ambiente extemo.

Asimismo, debemos también considerar la implementación del Sistema de Reestructuración Patrimonial, el cual tiene más de siete años de vigencia y por el cual han pasado empresas de todos los sectores económicos; de diferentes tamaños, dis-

\footnotetext{
- Director Encargado del Instituto de Investigación de Ciencias Financieras y Contables y Docente
} Auxiliar de la Facultad de Ciencias Contables de la UNMSM. 
tinto número de trabajadores, variados niveles de endeudamiento y también con variadas razones para ingresar a este sistema ya sean estos problemas coyunturales o estructurales.

El Sistema de Reestructuración Patrimonial, con la intervención de INDECOPI, en primer lugar es una herramienta eficaz para las empresas calificadas viables, y en segundo lugar busca una salida ordenada del mercado o la liquidación de otras que no pueden encontrar la supervivencia del negocio.

\section{La Crisis Empresarial}

Cuando una empresa tiene dificultades derivadas por diversos factores, ya sean internos como externos, se encuentra en una crisis empresarial, ésta puede involucrar el riesgo de pérdidas sustanciales tanto para los miembros de la propia organización como para los trabajadores que pueden perder sus empleos, para los inversores que han inver- tido su capital; además, de aquellas perscrem o entidades que como los proveedores th otorgado crédito, los clientes que han axelantado fondos, el Estado que deja de conimpuestos, los prestamistas, etc.

Estamos comentando un problema $x$ suma importancia que debe ser solucionaa: a nivel macroeconómico a través de política económicas adecuadas bajo un entorno empresarial competitivo, y un sistema jurídic : confiable. Sin embargo, desde el punto de vista interno, necesitamos aprender cómc manejar empresas en crisis agudizada por problemas de recesión económica donde los recursos se tornan escasos y no existe crecimiento, sino declinación de la economia peruana ya que éstas pueden volverse más frecuentes o prolongados. A la luz de estas consideraciones vale la pena investigar puesto que deseamos manejar adecuadamente la crisis empresarial para evitarla o por lo menos reducir su frecuencia de ocurrencia.

\section{CAUSAS CONORIGEN EXTERNO:}

\section{DEMERCADO}

- Competencia excesiva

- Fuerte caída de la demanda

DEL ENTORNO ECONÓMICO-SOCIAL-POLÍTICO

- Fase depresiva del ciclo

- Crisis sobrevenida (crisis política, crisis financiera de países y mercados, conflictos locales)

- Política económica del Gobierno

- Cambios sociales radicales y significativos

\section{CAUSAS CONORIGEN INTERNO:}

- Ineficacia de la dirección y/o gerencia

- Estrategias erróneas $o$ inadecuadas

- Sistema productivo ineficiente

- Inversiones improductivas

- Excesivo endeudamiento, agravado con el costo financiero elevado

- Fracaso de empresas del grupo económico

- Problemas concursales no resueltos

- Alta morosidad

\section{CAUSAS ESPECIALES:}

- Empresas se constituyen en mayor porcentaje de tipo familiar

- Nuevas empresas: El efecto de la globalización saca del mercado a empresas nuevas y de poco capital 
En el siguiente cuadro mostraremos las causas más habituales de la crisis empresarial.

Del cuadro anterior podemos comentar que la crisis empresarial puede tener su origen en numerosos y variados factores, por lo que nuestro enfoque investigador debe orientarse, en primer lugar, a establecer los problemas de la crisis en la información económica y financiera de la empresa identificando, a través de un primer análisis, los síntomas de insolvencia financiera tales como:

- Pérdidas operativas

- Flujos de caja negativos

- Endeudamiento alto y mal estructurado

- Carga financiera excesiva

- Fondos propios insuficientes

- Fondo de maniobra negativo

- Incumplimiento y no renovación de deudas a su vencimiento

La empresa, cuando está en crisis, muestra dos características que deben analizarse para tomar decisiones estratégicas en busca de recuperar y mejorar lo indicado anteriormente:

La amenaza, los participantes en una crisis sienten que no podrán lograr, obtener o mantener los valores, los recursos u objetivos que se visualizan como importantes. Estas amenazas pueden ser de naturaleza personal, organizacional o social. La amenaza comprende, por lo tanto, la magnitud o el valor de la pérdida potencial, así como la probabilidad de su ocurrencia; también comprende la magnitud o el valor de la pérdida mayor.

La Presion de Tiempo, es la percepción, por parte de los participantes de la crisis, de la cantidad de tiempo que tienen para buscar, deliberar y actuar antes que empiecen a ocurrir las pérdidas, es decir, es
Ta cantdadtde-tiempe-disponible para la acción.

\section{Las Tres Fases de la Crisis Empresarial}

Antes de entrar al análisis de las tres fases de la crisis empresarial debemos comentar que toda organización empresarial debe manejar adecuadamente el concepto de competitividad ya que dependiendo del nivel de ésta, la empresa entrará en crisis o no. La competitividad es la capacidad de una organización pública o privada, lucrativa o no, de mantener sistemáticamente ventajas comparativas que le permitan alcanzar, sostener y mejorar una determinada posición en el entorno socioeconómico; entonces una organización, cualquiera que sea la actividad que realiza, si desea mantener un nivel adecuado de competitividad a largo plazo debe utilizar, antes o después, unos procedimientos de análisis y decisiones formales, encuadrados en el marco del proceso de planificación estratégica. La función de dicho proceso es sistematizar y coordinar todos los esfuerzos de las unidades que integran la organización encaminados a maximizar la eficiencia global.

Para explicar mejor dicha eficiencia consideremos los niveles de competitividad, tanto interna como la externa. La competitividad interna se refiere a la capacidad de organización para lograr el máximo rendimiento de los recursos disponibles, como personal, capital, materiales, ideas, etc., y los procesos de transformación. Al hablar de la competitividad interna nos indica que la empresa ha de competir contra sí misma, como expresión de su continuo esfuerzo de superación.

La competitividad externa está orientada a la elaboración de los logros de la organización en el contexto del mercado, 0 del sector a cual pertenece. Como el sistema de referencia o modelo es ajeno a la em- 
presa, ésta debe considerar variables exógenas como el grado de innovación, el dinamismo de la industria, la estabilidad económica para estimar su competitividad a largo plazo. La empresa, una vez alcanzado un nivel de competitividad externa, deberá disponerse a mantener su competitividad futura basada en generar nuevas ideas y productos y de buscar nuevas oportunidades de mercado.

Cuando una empresa va perdiendo competitividad, entonces debe ir modificándose la administración del negocio ya que las empresas no mueren de infarto sino de inanición y que esta anemia es causada por la gran capacidad del empresario de autoengañarse, tal como lo manifiesta Pedro Nueno en su obra Reflotando la Empresa.

El análisis de las tres fases de la crisis empresarial nos servirá como asesores de empresas a identificary manejar el problema ya que la oportunidad de su determinación es importante por las características que debemos manejar en el planeamiento estratégico de la empresa ha reflotar como son la amenaza y presión del tiempo que podría conducirnos a un nivel de insolvencia irreparable.

\section{Fase I: Indicios o Síntomas}

Esta etapa, generalmente, se inicia luego de un plan de expansión con un alto nivel de endeudamiento, es decir, sin autonomía financiera. La imagen de éxito y prosperidad facilita los créditos que modifican sustancialmente la relación deuda/capital; de este modo, la empresa no tiene reservas ni se halla preparada para una etapa prolongada de crisis.

Los primeros indicios de la recesión empresarial se presentan con una reducción súbita de las ventas y con ello la acumulación de inventarios. En esta etapa, la dirección debe evaluar si se trata de un bache en el camino o son los primeros síntomas de la regresión. Si se dejan pasar estas señales y no se adopta programa alguno las consecuencias pueden ser graves.

Así, todo empresario con años de éxito niega la realidad del freno y por ello no reconoce el proceso. Normalmente, se resiste a aceptar la realidad impuesta por la competencia y el mercado negándose a adoptar una política de contracción más eficaz. Con ello se olvida que así como la economía se expande y se contrae, también esos ciclos afectan su mercado y no está en sus manos evitarlo.

\section{Fase II: Intensificación de los Problemas}

Se presenta una regresión continuada y agravada, en esta fase se tienen que originar cambios en los objetivos gerenciales como el mantener un flujo de efectivo adecuado a las operaciones de la empresa ya que tener efectivo es como mantener la sangre para el ser humano y sin ello la empresa entrará en una quiebra financiera aun cuando tenga activos adecuadamente valuados.

Con la caída de la producción por falta de ventas se presentarán pérdidas constantes, por lo que no debemos olvidar que lo más urgente en toda reestructuración es recuperar el efectivo ya que los prestamistas podrán ser comprensivos con los préstamos ya otorgados; pero, renuentes a otorgar nuevos créditos "nadie quiere poner dinero bueno sobre dinero malo". Por ello la necesidad de liquidez nos obligará a tomar acciones como: vender instalaciones esenciales para la generación de liquidez, hipotecar el futuro de la empresa vendiendo sus divisiones rentables, recortar los proyectos sobre el desarrollo de nuevos productos, $y$ dejar de mantener aspectos burocráticos 
derivados de las leyes laborales vigentes en el pais.

En la puesta en práctica de la reestructuración se encuentran varios problemas:

- La gerencia debe concentrarse en el activo del balance o inversión rentable y, sobre todo, evaluar constantemente el resultado de explotación derivada de dicha inversión ya que si el negocio no es viable en mérito a su propia actividad económica no vale la pena continuar operando.

- Se debe definir el alcance, ritmo e intensidad de la contracción empresarial (downsizing), los autores modernos hablan del righsizing pues en cada industria hay un tamaño adecuado debajo del cual mejor es salir porque no se tiene oportunidad de competir.

- Se debe proceder a la enajenación de todos aquellos elementos, unidades o líneas de producción, no necesarios o poco productivas para lo cual se debe analizar cada uno de ellos por el margen de contribución que reportan en la generación de la rentabilidad de la empresa en crisis.

- Proceder a la enajenación de aquellos elementos rentables que significan ingreso de fondos sustanciales de efectivo, ya que la meta probablemente es agilizary lograr flujo de efectivo para no cerrar la empresa. Es decir, es mejor perder algo valioso que aferrarse a él y perderlo todo.

- Muchas veces el cierre de instalaciones y la integración de la producción absorben fondos más altos que los que libera. Un ahorro mal diseñado, en relación al personal, puede generar mayores costos en mermas de materias primas y reclamos de clientes. Por ello, cada medida debe ser analizada mediante el principio económico básico del costo-beneficio.
- Cuando se obtiene efectivo por la venta de activos, esta debe ser para pagar nuevas iniciativas que requiere la reestructuración a través de la aplicación de estrategias; caso contrario, se estará liquidando la empresa en forma ordenada. Sin embargo, bajo este esquema debemos manejar adecuadamente el conflicto que se crea entre la empresa y los prestamistas ya que estos últimos requerirán que se les cancele la deuda pendiente y los primeros priorizarán sus estrategias de reestructuración.

En esta segunda fase debemos implementar un Programa de Reestructuración Empresarial que conduzca a la empresa, en crisis, a una reestructuración integral a nivel estratégico, organizativo y financiero.

Para que la reestructuración de la empresa en crisis sea viable es fundamental que el reestructurador cuente con un Plan General aceptado por la totalidad de los acreedores, ya que es importante salir airosos de la crisis que estemos enfrentando.

\section{Fase III: Incapacidad de Detener el Deterioro}

En esta fase, la situación del negocio continúa deteriorándose, por ello se debe optar por la decisión de acudir a la salida legal dispuesta en el Decreto Legislativo $N^{\circ} 845$ y optar por una de las siguientes alternativas:

- Reestructuración por insolvencia

- Liquidación

- Quiebra

Este tema es materia de análisis individualizado por parte de cada empresa en crisis que acude al INDECOPI, ya que en la realidad peruana estas alternativas se presentan como: 
Procedimiento de Insolvencia: Es una alternativa utilizada para el deudor como para el acreedor, en caso del deudor debe acreditar que más de las dos terceras partes del total de sus obligaciones están impagas por más de treinta días, o que tiene pérdidas acumuladas, deducidas las reservas, cuyo importe sea mayor de las dos terceras partes del capital social pagado. Los acreedores, por su parte, deben acreditar que se les adeuda un crédito mayor a 50 UIT vigentes a la fecha de la solicitud. En este procedimiento los acreedores pueden optar por aprobar el Plan de Reestructuración o decidir la liquidación de la empresa.

Procedimiento transitorio: La decisión gira en torno a la decisión de aprobación de un convenio de saneamiento y, de no aprobarse, se remite todo a la Comisión de Reestructuración Patrimonial para que ésta declare la insolvencia de oficio y los acreedores puedan tomar la decisión que corresponda.

Procedimientos preventivos: Entre éstos están el concurso preventivo y procedimiento simplificado que son mecanismos de refinanciación o reprogramación de pagos de deudas que se pueden iniciar a pedido de cualquier deudor a nivel nacional. Estos mecanismos buscan prevenir situaciones de insolvencia, ya que resulta más eficiente y menos costoso prevenir la crisis antes que tratarla una vez que ésta se haya presentado.

\section{Reestructuración Empresarial}

Las empresas, como las personas, pueden encontrarse sanas y luego caer enfermas o en crisis. En el caso de las personas, éstas visitan a un médico que los somete a diversas pruebas y les recomiendà un tratamiento. En el caso de las empresas es análogo, el gerente o dueño visita a un reestructurador que le recomienda una reformulación formal y lo monitorea hasta sacarlo de la crisis en que se encuentra la empresa.

Las empresas, en una economía capitalista, tienen que generar utilidades si quieren sobrevivir, ya que si éstas quiebran y dejan de operar por ineficiencia es innato y esencial al sistema capitalista. El profesor Jean Paul Sallenave dice que hay tres palabras en el pensamiento de un gerente o due-

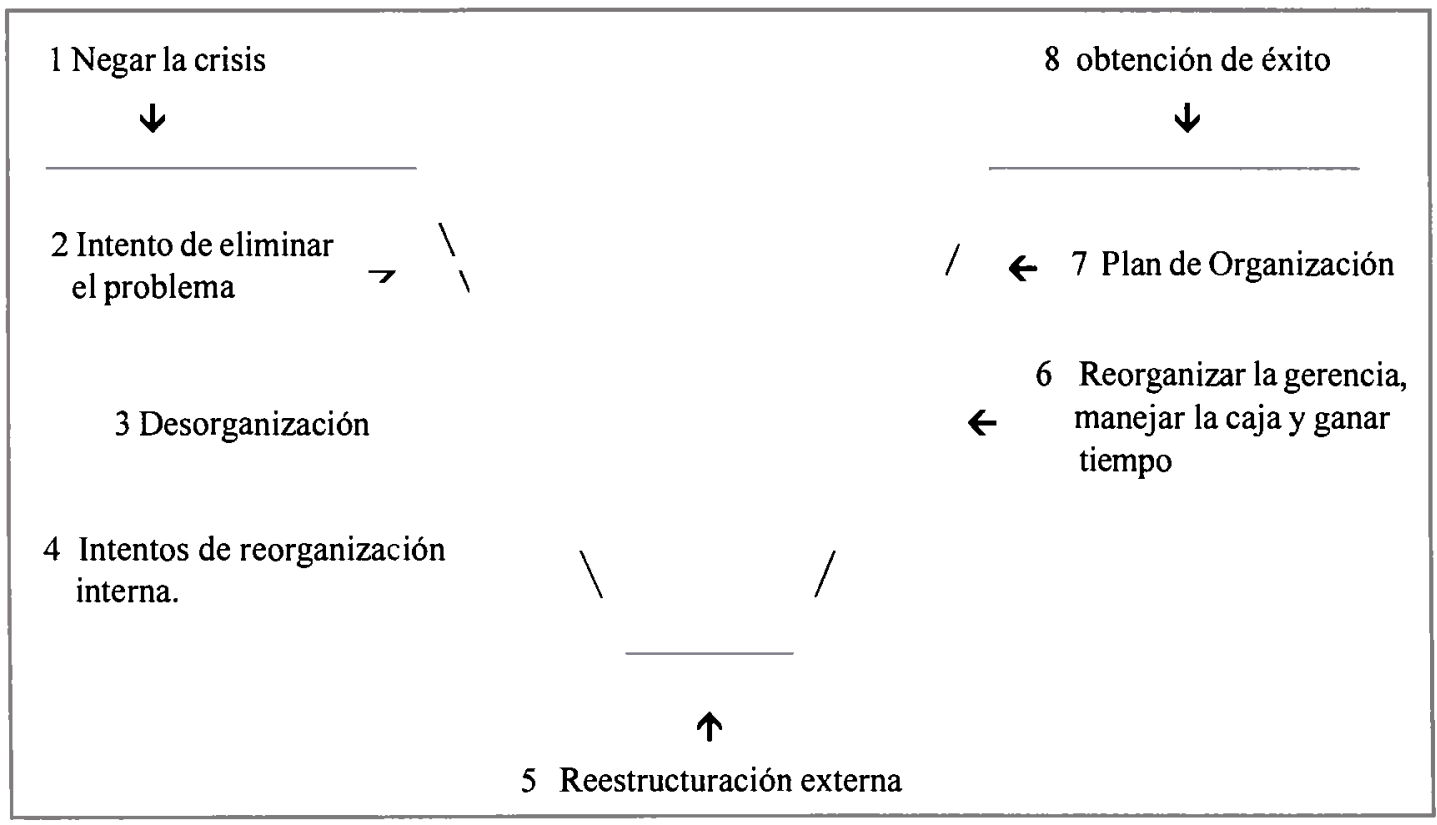


ño: Supervivencia, crecimiento y utilidad; la coyuntura económica fluctúa, los gerentes van y vienen; pero, la empresa tiene que enfrentar esas tres alternativas para superar la crisis.

Las etapas por las que atraviesa una empresa en crisis y que se ajustan a ella se pueden observar en el siguiente gráfico:

Al principio, se niega la crisis, luego se intenta resolverla internamente y se cae en desorganización para convencerse de que muchas veces se requiere de ayuda externa. Es difícil ser uno mismo su propio cirujano. El reestructurador tiene que reorganizar la gerencia, manejar la caja, renegociar con los acreedores, etc. Con miras a ganar tiempo, preparar un plan de reestructuración para reflotar la empresa, obtener éxito y cambiar el rumbo de la empresa.

En el plan de reestructuración es necesario, primero, tener un diagnóstico claro, preciso y rápido del problema. Es preciso saber dónde estas y adonde vas. Sallenave y otros autores hablan de siete factores de supervivencia:

1) Macroeconómico: Inflación recesión, devaluación, interés, aranceles, etc.

2) Producto: Estándar o diferenciado, único o diversificado.

3) Mercado: tamaño, tendencia, competitivo o monopólico.

4) Tecnología: avance, obsolescencia.

5) Competencia: externa o interna.

6) Capital: subcapitalizado, endeudamiento, capital de trabajo, etc.

7) Personal: gerencia, rotación de personal, capacitación, moral.

Sobre estos factores se debe considerar tres tipos de acciones: a) Refuerzo: atenuar la causa de las debilidades del negocio.

b) Redespliegue: migrar a otros mercados y productos donde probablemente se pueda ser competitivo.

c) La acción política: presión a gremios y al público.

Existen varios tipos de reestructuraciones tales como: la estratégica, la organizativa, procesal y financiera. Estas reestructuraciones están ligadas entre sí y es muy probable que se requieran varias, o todas a la vez, para reestructurar una empresa. Sin embargo, como nuestro objetivo es "darle la vuelta" a una empresa, pasando de una etapa de crisis a una de reflotamiento o de indicadores financieros de rojo a azul, tales como:

- Rentabilidad: margen operativo, utilidad neta, etc.

- Solvencia: grado de propiedad, palanqueo.

- Liquidez: flujo de caja neto, relación corriente, grado de propiedad corriente.

De los indicadores mencionados la liquidez requiere de una atención preponderante por parte del reestructurador ya que "la liquidez es para la empresa como la sangre para el ser humano"; es decir, sin ella no hay sobrevivencia. Cuando una empresa pierde caja a diario requiere de estrategias para gerenciar la crisis, para ello se debe considerar, entre otras, cinco elementos claves:

a) Seleccionar un equipo para gerenciar la crisis

b) Vender activos para levantar la caja

c) Reestructurar deudas

d) Cortar gastos y

e) Levantar la moral del grupo 


\section{Consideraciones Relevantes para un Proceso de Reestructuración Empresarial}

a) La empresa en crisis no debe gastar más de lo que tiene o genera por su nivel de operatividad normal.

b) En el Plan de Reestructuración se debe precisar dónde y cómo está la empresa, y adonde va después del proceso.

c) Recuerde que una empresa quebrada no vale nada.

d) Tener presente que desde el punto de vista financiero los pasivos son ciertos $y$ los activos son inciertos.

e) Cuando se plantee una decisión o estrategia, considere que es mejor obtener un porcentaje de algo que $100 \%$ de nada porque las oportunidades más importantes no aparecen más de una vez.

f) Los empresarios deben manejar estados financieros con cifras razonables que reflejen su realidad financiera, es decir, buscar el sinceramiento de sus valores contables ya que la peor muerte es por terquedad y desconocimiento de su propia realidad.

\section{Principios Básicos en un Proceso de Reestructuración Empresarial}

En una reestructuración empresarial cinco son los principios básicos que se deben aplicar:

- Reducción de costos

- Reestructuración de deudas

-Recapitalización

- Gerencia profesional

- Contar con un Plan Estratégico

De los cinco principios mencionados comentaremos el primero de ellos ya que
Reducir Costos es tan importante que amerita estudiarlo y analizarlo para responder la pregunta, ¿cómo hacerlo?, para ello plantearemos las siguientes sugerencias:

a) Adoptar una filosofía de reducción de costos, esto no sólo para una empresa en crisis; sino también para una empresa en marcha, ya que los sobrecostos siempre ocasionan disminución en los niveles de competitividad y de ventas. Sin embargo, en el largo plazo es más importante incrementar las ventas que reducir costos.

b) La empresa debe conocer sus costos ya que la identificación es clave para evitar la disminución indiscriminada que puede ser peligrosa.

c) Concentrar los esfuerzos de reducción de costos en aquellas categorías que generan mayor gasto considerando la Ley de Pareto (80/20) que indica que el $20 \%$ de las actividades generan $80 \%$ de los resultados; $80 \%$ de los costos provienen de $20 \%$ de las actividades. En conclusión, 80\% del desperdicio proviene del $20 \%$ de las categorías de gasto.

d) La reducción de costos es una tarea de día a día, para esto la gerencia debe cortar costos en activos innecesarios, inventarios de rotación lenta, clientes marginales, gastos innecesarios, etc.

e) No se debe cortar costos relacionados con el incentivo a la productividad del negocio, las ventas y compras de activos productivos.

f) Contar con indicadores para medir el valor agregado por trabajador, eliminar desperdicio de tiempo y contener costos creando comités de costos a plazo fijo y mandatos claros. 
g) Simplificar la oferta de productos y servicios e incrementar la velocidad para reuucir costos aumentando la productividad e incrementando la rotación de los inventarios.

h) Crear confianza con los proveedores más importantes para obtener mejores orecios y repartos que aminoren los costos.

i) Outsourcing para ahorrar dinero, capitalizando las ventajas de otras compañías en el mercado se puede outsource los servicios de soporte de la empresa tales como la contabilidad, el mantenimiento de a tecnología de la información, etc.

j) Mantener el número de trabajadores a niveles reducidos, eliminando los improsuctivos, y buscando medir la productividad se los más eficientes para compararlos en el iempo y con otras empresas.

k) Reingenierías y reestructuraciones, se debe tomar en cuenta que el $70 \%$ de las reingenierías fallaron al olvidar que en los negocios no se busca una "eficiencia suprema"; sino buscar reestructurar el trabajo que se realiza en una empresa sin eliminar empleos, es decir, buscar ser una organización virtual.

1) Manejar y crear presupuestos para todo y mantenerse en él.

\section{CONCLUSIONES}

- En los últimos años muchas empresas han entrado en crisis debido a los problemas de liquidez ocasionados por diversos factores como el enfriamiento de la economía mundial y la recesión interna que ha afectado no sólo la rentabilidad, que es una variable fundamental de supervivencia empresarial; sino, también la disminución de los niveles de producción y ventas de bienes y servicios, aspectos que conllevan a la crisis empresarial y que provoca la disminución del tamaño de la inversión del negocio y probable despido de personal, lo cual es un problema social primordial a resolverse en un país subdesarrollado como el nuestro.

- Nuestro objetivo general es elaborar un estudio para gestionar empresas que se encuentran en crisis, y el objetivo específico es mencionar los mecanismos de acceso y salida del mercado de las empresas viables que se encuentran en estado de reestructuración patrimonial; en los casos que no se pueda reestructurar, optar por la liquidación de empresas. La gestión financiera de empresas en crisis, se basa en las doctrinas y teorías de manejo empresarial, recomendando la elaboración de un Plan Estratégico para las empresas en crisis, tomando en cuenta que la gestión se enfrenta constantemente a la trilogía: Supervivencia, crecimiento y utilidad.

- Cuando se trabaja en una empresa en crisis el problema es detener la caída e iniciar la recuperación, por lo que implica un doble frente de actuación:

* La reestructuración estratégica, que implica identificar las deficiencias y debilidades de las empresas en crisis, las medidas que se deben adoptar para subsanarlas y la ejecución de planes que demanden importantes recortes y reducción drástica de la dimensión empresarial para recuperar la rentabilidad.

* La reestructuración financiera tendrá como objetivo mantener a raya a los numerosos acreedores, preservar y reducir la carga del servicio de la deuda, aumentar la liquidez, convertir de ser posible la deuda en capital, buscar un consenso entre los acreedores para no liquidar la empresa; esto debido a ya que los efectos sociales y econó- 
micos son graves en un país como el nuestro donde lo fundamental es la generación de puestos de trabajo a través de empresas competitivas que ayuden al desarrollo del pais.

- Por otro lado, la utilización de la información contable es aún empírica por prepararse más para fines tributarios que financieros, aspecto que no permite obtener una información cuantificable objetiva y sistemática de la realidad económica y financiera de la empresa; es decir, los reportes financieros no expresan el sinceramiento de los valores razonables de los negocios, los mismos que distorsionan los ratios e indicadores calculados, limitando su utilización cuando se pretende evaluar e interpretar la información contable disponible para ofrecer un diagnóstico de la empresa sobre su situación pasada, presente y futura.

- En la realidad peruana las empresas aún no han normalizado, con mayor o menor éxito, un modelo de diagnóstico económicofinanciero utilizado por el analista para la toma de decisiones tanto en el ambiente interno como externo. Hoy en día, el alto índice de morosidad que arroja el sistema financiero conlleva que las técnicas de predicción o evaluación del riesgo sean lo más objetivas y certeras posibles; en este sentido, el presente trabajo busca desarrollar un marco conceptual que permita abordar convenientemente la teoría económica de la insolvencia.

- El empresario debe entender que todo análisis estratégico tiene al menos tres dimensiones a conocer: el producto, la industria (sector) y la empresa (entorno). Entonces, el análisis de la crisis comprenderá, entre otras, las acciones en torno a las cuales se implementan los procedimientos de gestión esencial para la supervivencia y recuperación; además de la identificación de los factores y problemas de la empresa la finalidad de aplicar las medidas corre. tivas correspondientes. 
1. IVANCEVICH J.M., Lorenzi Peter, Crosby Philip.

1996. Gestión, Calidad y Competitividad. Editorial Irwin.

2. THORNE, Paul. 1997. El nuevo Gerente General. Colombia, Editorial McGraw-Hill.

3. MANRIQUE R. Francisco. 1997. Un Cambio de Epoca, no una Epoca de Cambios. La Gerencia Latinoamericana debe Cambiar. Colombia, Editorial McGraw-Hill.

4. DRUCKER, Peter.

1996. La Gerencia del Futuro. Colombia, Editorial Norma.

5. YIP, George S. 1993. Globalizacion Estrategias para Obtener una Ventaja Competitiva Internacional. Editorial Norma.

6. STONER, James R.F. 1996. Administración. México, Editorial Prentice Hall Hispanoamericana.

7. HAMMER, Michael \& Champy James. 1996. Reingenieria. Grupo Editorial Norma.

8. DE COSSIO, José Luis.

1995. Crisis Empresarial. Ediciones Cossio. Lima.

9. MUÑOZ, Estuardo.

1997. Reestructuración Patrimonial. Editorial Rhodas, Lima

10. INDECOPI.

1996. Recopilación Ordenada de las Leyes del INDECOPI. Editorial del INDECOPI.
11. NUENO, Pedro. 1991. Reflotando la Empresa. Ediciones Deustuo S.A.

12. ORTER, Michael. 1987. Ventaja Competitiva. Compañía Editorial Continental S.A., México.

13. SENGE, Peter.

1998. La Quinta Disciplina. Editorial Granica, Mexico.

14. INDECOPI. Seis Experiencias Empresariales de Reestructuración Patrimonial, Perú 2000

15. BOLOÑA BERH, Carlos. Experiencias para una Economía al Servicio de la Gente, Perú 2000. 